\title{
A DECOMPOSITION THEOREM FOR CLOSED COMPACT CONNECTED P.L. $n$-MANIFOLDS
}

\author{
B. G. CASLER
}

\begin{abstract}
Let $M$ be a compact connected P.L. $n$-manifold without boundary. Then $M$ is the union of 3 sets $E_{1}, E_{2}$ and $F$ where $E_{i}, i=1,2$, is homomorphic to the interior of an $n$-ball and $F$ is the P.L. image of an $(n-1)$-sphere. Further each point of $F$ is a limit point of $E_{1}$ and $E_{2}$.
\end{abstract}

It is well known that an $n$-sphere is the union of three disjoint sets $E_{1}$, $E_{2}, F$ where $E_{1}$ and $E_{2}$ are topologically equivalent to an Euclidean $n$-space and $F$ is topologically equivalent to an $(n-1)$-sphere. The set $F$ can be thought of as an embedded (n-1)-sphere in an $n$-sphere. In this note it will be shown that every compact connected $n$-manifold has a very similar property. Namely,

THEOREM. Every compact connected P.L. n-manifold, without boundary $n>0$, is the union of three disjoint sets $E_{1}, E_{2}$ and $F$ where

(i) $E_{i}$ is topologically equivalent to $E^{n}$,

(ii) $F$ is the P.L. image of $S^{n-1}$,

(iii) each point of $F$ is a limit point of $E_{i}, i=1,2$.

Proof. Let $M$ be a closed compact connected combinatorial $n$-manifold of dimension $\geqq 3$. (The theorem is trivial for dimension 1 and an obvious modification of the proof for dimension $\geqq 3$ will yield a proof of the theorem for dimension 2.) Let $T$ be some fixed triangulation that is the second barycentric subdivision of some triangulation of $M$. Choose some $n$-simplex $\sigma$ in $T$. It was shown in [1] that there is a spine $K$ of $M \backslash \operatorname{Int}(\sigma)$ such that

(i) the closure of each component of each intrinsic skeleton is a combinatorial cell triangulated by the 3 rd barycentric subdivision, $T^{3}$, of $T$,

(ii) if $p$ is a vertex of $K$, then the closure of each component of $\operatorname{St}(p, M) \backslash K$ is a cell,

(iii) $K$ is of dimension $(n-1)$ at each point of $K$.

Received by the editors April 9, 1971.

AMS 1970 subject classifications. Primary 57C05.

Key words and phrases. P.L. manifold, spine, decomposition.

(c) American Mathematical Society 1972 
Let $C_{(n-1), j}$ and $C_{(n-2), j}$ be the components of the intrinsic $(n-1)$ skeleton and the intrinsic $(n-2)$-skeleton of $K$ respectively. If $C_{(n-2), j} \subset$ $\mathrm{Cl}\left(C_{(n-1), i}\right)$, let $A_{i, j}$ be a polyhedral arc from a point $p_{i} \in \operatorname{Int}\left(\mathrm{Cl}\left(C_{(n-1), i}\right)\right)$ to a point of $q_{j} \in \operatorname{Int}\left(\mathrm{Cl}\left(C_{(n-2), j}\right)\right)$. Since dimension of $M \leqq 3$, it may be assumed that $\operatorname{Int}\left(A_{i, j}\right) \cap \operatorname{Int}\left(A_{r, s}\right)=\varnothing$ if $i \neq r$ or $j \neq s$ and that $\operatorname{Int}\left(A_{i, j}\right) \subset$ $\operatorname{Int}\left(\mathrm{Cl}\left(C_{(n-1), i}\right)\right)$. Let $H^{\prime}$ be a maximal tree in the union of the $A_{i, j}$ 's. Let $H \subset H^{\prime}$ be a subtree such that if $q_{j} \in H, q_{j}$ is of order at least 2. For each $q_{j} \in H$, let $\operatorname{St}\left(q_{j} M, T^{5}\right)=B_{j}$. Because of properties of $K, B_{j} \cap \mathrm{Cl}\left(C_{(n-1), i}\right)$ is either an $(n-1)$-cell or empty. If $\left(B_{j} \cap C_{(n-1), i}\right) \cap H$ contains an arc $A$ such that $q_{j} \in A$ then call $\mathrm{Cl}\left(B_{j} \cap C_{(n-1), i}\right)$ a good cell. Otherwise call $\mathrm{Cl}\left(B_{j} \cap C_{(n-1), i}\right)$ a bad cell.

Let $K^{\prime}$ be $K$ with the interiors of all bad cells deleted. Since $\mathrm{Cl}\left(B_{j} \cap C_{(n-1), i}\right)$ is a cell, the closure of each component of the intrinsic $(n-1)$-skeleton of $K^{\prime}$ is a cell. Let $K^{*}$ be the union of all the boundaries of all the closures of the components of the intrinsic $(n-1)$-skeletor of $K^{\prime}$. Let $L=\mathrm{Cl}\left(K^{\prime} \mid N\left(K^{*}, M, T^{5}\right)\right)$. $\left(N\left(K, L, T^{i}\right)\right.$ denotes the union of the closed simplices of $L$ triangulated by $T^{i}$ that have a vertex in $K$.) Since $L$ is the union of $(n-1)$-cells $L \cup H$ collapses to $H$. Let $\left(\bigcup B_{i}\right) \cup N\left(L, M, T^{5}\right)=Q$. By construction $M \backslash \operatorname{Int}(\sigma)$ collapses to $Q$. Since the neighborhood was taken in the 5th barycentric subdivision each point of $K^{*}$ is accessible from $\sigma$. Hence the point set boundary of $Q$ is the P.L. image of $S^{n-1}$ and $M \backslash Q$ is homeomorphic to $E^{n}$. Since $L \cup H$ is collapsible, $\operatorname{Int}(Q)$ is homeomorphic to $E^{n}$ and the theorem follows.

\section{REFERENCES}

1. B. G. Casler and T. J. Smith, Standard spines of compact connected combinatorial n-manifolds with boundary (to appear).

Department of Mathematics, louisiana State University, Baton Rouge, LOUISIANA 70803 